\title{
TWO OBSERVATIONS ON THE CONGRUENCE EXTENSION PROPERTY
}

\author{
G. GRÄTZER AND H. LAKSER ${ }^{1}$
}

\begin{abstract}
A pair of algebras $\mathfrak{A}, \mathfrak{B}$ with $\mathfrak{B}$ a subalgebra of $\mathfrak{U}$ is said to have the (Principal) Congruence Extension Property (abbreviated as PCEP and CEP, respectively) if every (principal) congruence relation of $\mathfrak{B}$ can be extended to $\mathscr{H}$. A pair of algebras $\mathfrak{A}, \mathfrak{B}$ is constructed having PCEP but not CEP, solving a problem of A. Day. A result of A. Day states that if $\mathfrak{B}$ is a subalgebra of $\mathfrak{U}$ and if for any subalgebra $\mathfrak{C}$ of $\mathfrak{U}$ containing $\mathfrak{B}$, the pair $\mathfrak{A}$, $\mathfrak{C}$ has PCEP, then $\mathfrak{A}, \mathfrak{B}$ has CEP. A new proof of this theorem that avoids the use of the Axiom of Choice is also given.
\end{abstract}

1. The example. Let $A=\{a, b, c, d, e, f\}$. We define a binary operation + on $A$ by $a+f=e, b+f=e, x+y=x$ otherwise. Let $\mathfrak{A}=\langle A ;+\rangle$ and $B=\{a, b, c, d\}$. Then $\mathfrak{B}$ is a subalgebra of $\mathfrak{i}$. An easy computation shows that $\mathfrak{A}, \mathfrak{B}$ has PCEP. Now let $\Theta=\Theta_{\mathfrak{B}}(a, c) \vee \Theta_{\mathfrak{B}}(b, d)$. Then $c \not \equiv d(\Theta)$. However, if $\bar{\Theta}$ denotes the smallest congruence of $\mathfrak{H}$ with $\bar{\Theta}_{B} \geqq \Theta$, then $a \equiv c(\bar{\Theta})$; hence $a+f \equiv c+f(\bar{\Theta})$, that is, $e \equiv c(\bar{\Theta})$. Similarly, $b \equiv d(\bar{\Theta})$, and so $e \equiv d(\bar{\Theta})$. By transitivity, $c \equiv d(\bar{\Theta})$. Thus $\bar{\Theta}_{13} \neq \Theta$. This means that the pair $\mathfrak{H}, \mathfrak{B}$ does not have CEP.

2. The proof. We want to prove the following important ${ }^{2}$

THEOREM (A. DAY [1]). Let $\mathfrak{Q}$ be an algebra and $\mathfrak{B}$ a subalgebra of $\mathfrak{A}$. If PCEP holds for any pair $\mathfrak{H}, \mathfrak{C}$ where $\mathfrak{C}$ contains $\mathfrak{B}$, then $\mathfrak{N}, \mathfrak{B}$ has CEP.

Our proof, as well as Day's, is based on the following (A. W. Goldie [3], see also G. Grätzer [4] and Exercise 64 of Chapter 1 in G. Grätzer [5]):

Lemma. Let $\mathfrak{A}$ and $\mathfrak{B}$ be algebras and let $\mathfrak{B}$ be a subalgebra of $\mathfrak{U}$. Let $\Phi$ be a congruence relation of $\mathfrak{A}$ and $\Theta$ be a congruence relation of $\mathfrak{B}$ satisfying $\Phi_{B 3} \leqq \Theta$. Set $D=[B] \Phi=\{x \mid x \in A, x \equiv y(\Phi)$ for some $y \in B\}$. We define

Received by the editors December 13, 1971.

AMS 1970 subject classifications. Primary 08A25; Secondary 08A15.

Key words and phrases. Universal algebra, congruence relation, Congruence Extension Property.

${ }^{1}$ The research of both authors was supported by the National Research Council of Canada.

${ }^{2}$ For an interesting application of this result see [2]. 
a binary relation $\Theta(\Phi)$ on $D$ by the rule $u \equiv v(\Theta(\Phi))$ iff $u \equiv x(\Phi), x \equiv y(\Theta)$, $y \equiv v(\Phi)$ for some $x, y \in B$. Then $\mathfrak{D}$ is a subalgebra of $\mathfrak{A}$ and $\Theta(\Phi)$ is a congruence relation on $\mathfrak{D}$. Furthermore, $(\Theta(\Phi))_{B}=\Theta$.

Proof of the Theorem. Let $\mathfrak{U}$ and $\mathfrak{B}$ be given as in the Theorem. We shall prove that, for any subalgebra $\mathfrak{C}$ of $\mathfrak{A}$ containing $\mathfrak{B}$, the pair $\mathfrak{A}, \mathfrak{C}$ has CEP. Let $\Theta$ be a congruence relation on $\mathbb{C}$ and let $\bar{\Theta}$ be the smallest congruence relation on $\mathfrak{A}$ satisfying $\bar{\Theta}_{C} \geqq \Theta$. Obviously,

$$
\bar{\Theta}=\bigvee\left(\Theta_{\mathcal{U}}(x, y) \mid x, y \in C \text { and } x \equiv y(\Theta)\right) \text {. }
$$

We want to show that

$\left(^{*}\right)$ for $a, b \in C, a \equiv b(\bar{\Theta})$ implies that $a \equiv b(\Theta)$

(this is CEP). In view of the formula for $\bar{\Theta}$ and the way joins of congruences can be described, $\left({ }^{*}\right)$ is equivalent to:

(**) For any subalgebra $\mathfrak{C}$ of $\mathfrak{A}$ with $B \subseteq C$, if $a, b \in C, a_{1}, b_{1}, \cdots, a_{n}$, $b_{n} \in C, a_{i} \equiv b_{i}(\Theta)$ for $i=1, \cdots, n, a=x_{0}, x_{1}, \cdots, x_{n}=b, x_{i} \in A$ for $i=1, \cdots, n-1$, and $x_{i-1} \equiv x_{i}\left(\Theta_{\mathscr{Y l}}\left(a_{i}, b_{i}\right)\right)$ for $i=1, \cdots, n$, then $a \equiv b(\Theta)$.

We prove this statement by induction on $n$. For $n=1$ it is obvious since $\mathfrak{A}, \mathfrak{C}$ has PCEP. Now assume that $n>1$ and that the statement is valid for $n-1$. Set $D=[C] \Theta_{\mathfrak{U}}\left(a_{n}, b_{n}\right), \Theta_{0}=\Theta_{\mathbb{C}}\left(a_{1}, b_{1}\right) \vee \cdots \vee \Theta_{\mathbb{C}}\left(a_{n}, b_{n}\right)$. Since PCEP holds for $\mathfrak{A}, \mathfrak{C}$, we have $\left(\Theta_{\mathfrak{H I}}\left(a_{n}, b_{n}\right)\right)_{C} \leqq \Theta_{0}$; hence we can form $\Psi=\Theta_{0}\left(\Theta_{\mathfrak{Q}}\left(a_{n}, b_{n}\right)\right)$ and it will satisfy $\Psi_{C}=\Theta_{0}$. Now observe that $\mathfrak{A}, \mathfrak{D}$, $a=x_{0}, \cdots, x_{n-1}, a_{1}, b_{1}, \cdots, a_{n-1}, b_{n-1}$, and $\Psi$ satisfy the assumptions of (**) with $n-1$, hence we can conclude that $a \equiv x_{n-1}(\Psi)$. Obviously, $x_{n-1} \equiv x_{n}(\Psi)$, hence $a \equiv b(\Psi)$. Since $a, b \in C$ and $\Psi_{C}=\Theta_{0} \leqq \Theta$ we conclude that $a \equiv b(\Theta)$, completing the proof of $\left({ }^{* *}\right)$. If we now let $\mathfrak{C}=\mathfrak{B}$ the theorem follows.

\section{REFERENCES}

1. A. Day, A note on the Congruence Extension Property, Algebra Universalis 1 (1971), 234-235.

2. E. Fried and G. Grätzer, A nonassociative extension of the class of distributive lattices. I, II, Notices Amer. Math. Soc. 18 (1971), 402, 548. Abstract \#71T-A47; \#71T-A62.

3. A. W. Goldie, The Jordan-Hölder theorem for general abstract algebras, Proc. London Math. Soc. (2) 52 (1950), 107-131. MR 12, 238.

4. G. Grätzer, On the Jordan-Hölder theorem, for universal algebras, Magyar Tud. Akad. Mat. Kutató Int. Közl. 8 (1963), 397-406. MR 29 \#4717.

5. —_, Universal algebra, Van Nostrand, Princeton, N.J., 1968. MR 40 \#1320.

Department of Mathematics, University of Manitoba, Winnipeg, Manitoba, CANADA 